\title{
(6) OPEN ACCESS \\ Stability of octreotide acetate decreases in a sodium bisulfate concentration-dependent manner: compatibility study with morphine and metoclopramide injections
}

\author{
Kouichi Tanabe, ${ }^{1,2}$ Junko Wada, ${ }^{1}$ Jun Ohkubo, ${ }^{3}$ Atsumi Nitta, ${ }^{1}$ Tomoaki Ikezaki, ${ }^{3}$ \\ Miyako Takeuchi, ${ }^{3}$ Aya Handa, ${ }^{3}$ Mai Tanaka, ${ }^{3}$ Nozomu Murakami, ${ }^{4}$ Tatsuhiko Kashii, ${ }^{2}$ \\ Hidenori Kitazawa ${ }^{3}$
}

${ }^{1}$ Department of Pharmaceutical Therapy \& Neuropharmacology, Faculty of Pharmaceutical Sciences, Graduate School of Medicine and Pharmaceutical Sciences, University of Toyama, Toyama, Japan

${ }^{2}$ Department of Medical Oncology, Toyama University Hospital, Toyama, Japan ${ }^{3}$ Department of Pharmacy, Saiseikai Takaoka Hospital Takaoka, Japan ${ }^{4}$ Department of General and Digestive Surgery, Himi Municipal Hospital of Kanazawa Medical University, Kanazawa, Japan

\section{Correspondence to}

Dr Kouichi Tanabe, Department of Medical Oncology, Toyama University Hospital, 2630 Sugitani, Toyama City, Toyama Prefecture 930-0194, Japan; tanabe@po2.nsknet.or.jp

Received 20 August 2014 Revised 1 December 2014 Accepted 5 December 2014 Published Online First 16 January 2015

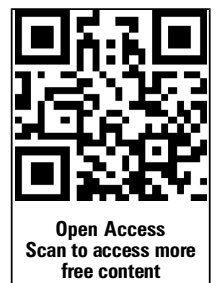

CrossMark

To cite: Tanabe K, Wada J, Ohkubo J, et al. Eur J Hosp Pharm 2015;22:171-175.

\section{ABSTRACT}

Purpose Sodium bisulfate is known to affect the stability of octreotide. However, the critical concentration of sodium bisulfate is not known. Therefore, we assessed the critical concentration of sodium bisulfate needed to preserve the stability of octreotide using actual drugs containing sodium bisulfate.

Methods Although morphine and metoclopramide preparations are considered to be compatible with octreotide, some of their products are known to contain sodium bisulfate. Thus, octreotide was mixed with preparations of sodium bisulfate solutions at serial concentrations and morphine and metoclopramide preparations containing sodium bisulfate, and octreotide stability was then evaluated using high performance liquid chromatography.

Results Octreotide concentrations decreased significantly at a sodium bisulfate concentration of $0.1 \mathrm{mg} / \mathrm{mL}$ or higher after 10 days when octreotide was mixed with sodium bisulfate solutions at various concentrations. A significant decrease in octreotide concentrations also occurred when it was mixed with morphine and metoclopramide preparations containing sodium bisulfate and stored for 10 days; however, slight decreases were observed in the mixture with both preparations and were within the clinically acceptable range for morphine preparations.

Conclusions These results indicate that the residual rate of octreotide decreases with time in a sodium bisulfate concentration-dependent manner when octreotide was mixed with morphine or metoclopramide. However, this incompatibility may be clinically acceptable when the final sodium bisulfate concentration is lower than $0.1 \mathrm{mg} / \mathrm{mL}$ and the mixed solution is used within 7 days.

\section{INTRODUCTION}

Carcinomatous peritonitis-induced gastrointestinal obstruction causes various digestive symptoms, such as nausea, vomiting and anorexia, which reduce the quality of life of patients. Octreotide (OCT) acetate is a somatostatin analogue that promotes the absorption of digestive juices and inhibits their secretion, and is also a key drug for treating digestive symptoms. ${ }^{1}{ }^{2}$ OCT is a cyclic synthetic octapeptide in which the retention of the S-S bond and cyclic structure is necessary for it to exhibit its bioactivity (figure 1)..$^{3-5}$ Because OCT is a peptide, it contains an amide bond that connects intramolecular amino acids and is readily hydrolysed. Hydrolysis was shown to be promoted in alkaline solutions, with an increase in the inactivation speed being reported in the presence of sodium bisulfate (SBS) as an additive. ${ }^{6-8}$ We previously investigated incompatibilities with dexamethasone (DEX) injections, which are sold as an ethical drug, and demonstrated that OCT concentrations were maintained for 10 days in a mixture with a DEX injection that did not contain SBS but were significantly decreased in a mixture with a SBS-containing DEX injection. ${ }^{9}$ However, the concentrations of SBS that have a clinical influence have not yet been determined, and the stability of these drugs, other than DEX, remains unclear.

Morphine (MOR) 1\% and 4\% are sold in the form of injections in Japan; however, SBS is used as a stabiliser in the $4 \%$ preparation only because the concentration of MOR hydrochloride is close to its solubility, $\quad 0.178 \mathrm{mM}$ (about $5.7 \% \quad(\mathrm{~W} / \mathrm{V})){ }^{10}$ Although metoclopramide (MET) injections generally contain SBS or other stabilisers (such as sodium citrate) as an additive to stabilise the $\mathrm{pH}$, this depends on the manufacturers. Previous studies have demonstrated that MOR and MET are compatible with OCT because no changes were observed in appearance, such as precipitation; however, the presence or absence of SBS as well as the hydrolysis of OCT were not described. ${ }^{11}$ Accordingly, the presence or absence of SBS and its concentration after mixing with OCT are considered to be very important factors in clinical studies evaluating the effects of OCT; they have been shown to directly influence the effects of OCT. In addition, the concentration of SBS that influences the stability of OCT has not been clarified, which presents difficulties when evaluating compatibilities with drugs containing SBS. Although MOR and MET are frequently used in combination with OCT for the palliative care of patients with end-stage cancer, ${ }^{12}{ }^{13}$ to the best of our knowledge, no study has investigated compatibility with a focus on the presence or absence of SBS. In the case of the patients who self-administer analgesic treatment by using a patient-controlled analgesia pump (PCA pump: balloon type or portable mechanical type), the elucidation of the drug combination stability in the PCA pump would minimise the number of venipunctures and the usage of consumables, such as medical cassettes. Therefore, it 


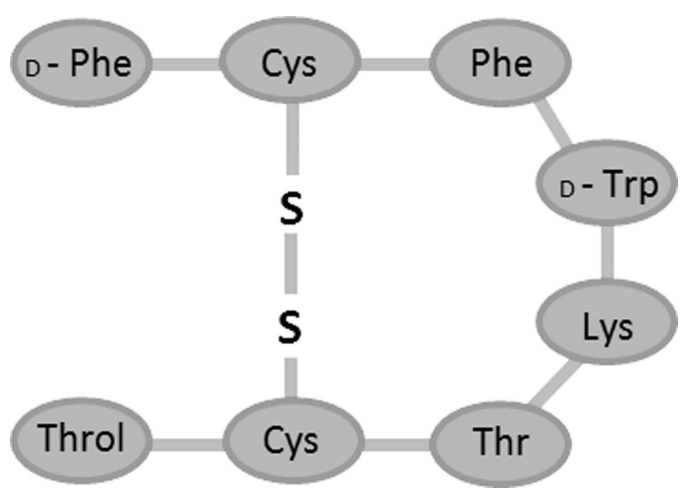

Figure 1 Structure of octreotide, an eight amino acid synthetic analogue of somatostatin.

is advantageous both economically and for the patient's quality of life. ${ }^{14-17}$

When aggressive treatment is not an option, OCT is an important drug that can be used to maintain the quality of life of patients until the end stage. ${ }^{12}$ However, large variations have been reported in the efficacy of OCT $(44 \%-100 \%) .{ }^{18}$ The time after the occurrence of intestinal obstruction, obstructed region, infusion volume and presence or absence of a nasogastric tube and concomitant antiemetic (such as MET and DEX) have already been proposed as factors that influence the effects of OCT; ${ }^{18}$ however, we hypothesised that this variation may be attributed to the SBS concentration. If this can be demonstrated, OCT can be used more effectively.

In the present study, we examined reproducibility, with a focus on the SBS concentration-dependent OCT stability of drugs made from MOR and MET, using high performance liquid chromatography (HPLC).

\section{METHODS}

\section{Materials}

OCT was obtained from Sigma-Aldrich Japan (Tokyo, Japan). SBS was obtained from Wako (Osaka, Japan). Sandostatin for subcutaneous injection ( $100 \mu \mathrm{g} / 1 \mathrm{~mL}$ ampoules) was purchased from Novartis Pharma (Tokyo, Japan). To obtain MOR products with different SBS concentrations, MOR hydrochloride injection $10 \mathrm{mg} / 1 \mathrm{~mL}$ ampoules (SBS free; 1\% MOR-SBS 0) and MOR hydrochloride injection $200 \mathrm{mg} / 5 \mathrm{~mL}$ ampoules (SBS $0.1 \mathrm{mg} / \mathrm{mL} ; 4 \%$ MOR-SBS 0.1 ) were purchased from Takeda Pharmaceutical (Tokyo, Japan). For MET products with different SBS concentrations, Elieten injection $10 \mathrm{mg} / 2 \mathrm{~mL}$ ampoules (SBS free; MET-SBS 0) and Primperan injection $10 \mathrm{mg} / 2 \mathrm{~mL}$ ampoules (SBS $1.09 \mathrm{mg} / \mathrm{mL}$ as sodium pyrosulfite $1 \mathrm{mg} / \mathrm{mL}$; MET-SBS 1.0) were purchased from Takata Seiyaku (Tokyo, Japan) and Astellas Pharma (Tokyo, Japan), respectively. Sodium pyrosulfite is a dehydrating condensation compound of binary molecular SBS, the actions of which are similar to SBS in aqueous solution. All other chemicals were commercial products of analytical reagent grade.

\section{Preparation of samples}

\section{Dependence on SBS concentration}

SBS was dissolved and diluted with saline. Sample solutions were collected from an OCT ampoule and mixed with SBS. The mixture was diluted with saline to adjust the final SBS concentration to $0,0.01,0.03,0.05,0.1,0.3$ and $0.5 \mathrm{mg} / \mathrm{mL}$ and the final OCT concentration to $41.7 \mu \mathrm{g} / \mathrm{mL}$, and $1 \mathrm{~mL}$ of each mixture was prepared. Each sample was added to a $1.5 \mathrm{~mL}$ microtube (Promega, Tokyo, Japan) and stored at room temperature with protection from light. OCT concentrations were then measured in samples containing SBS at various concentrations after $0,3,7$ and 10 days using HPLC.

\section{Compatibility study with MOR preparations}

Based on the actual regimen, the experiment was performed on the assumption of the 7-day administration of daily doses of OCT and MOR at $300 \mu \mathrm{g}$ and $30 \mathrm{mg}$, respectively. ${ }^{16}$ The necessary volumes were sampled from OCT and MOR ampoules and diluted with saline to a total volume of $50.4 \mathrm{~mL}$. The final OCT and MOR concentrations were $41.7 \mu \mathrm{g} / \mathrm{mL}$ and $4.17 \mathrm{mg} / \mathrm{mL}$, respectively. Two mixtures with $1 \%$ MOR-SBS 0 and $4 \%$ MOR-SBS 0.1 were prepared. The final SBS concentrations were 0 and $0.01 \mathrm{mg} / \mathrm{mL}$, respectively. A total of $1 \mathrm{~mL}$ was transferred from each mixture to a $1.5 \mathrm{~mL}$ microtube and stored at room temperature with protection from light. The OCT concentration in each sample was then measured after 3, 7 and 10 days using HPLC.

\section{Compatibility study with MET preparations}

Based on the actual regimen, the experiment was performed on the assumption of the 7-day administration of daily doses of OCT and MET at $300 \mu \mathrm{g}$ and $20 \mathrm{mg}$, respectively. ${ }^{16}$ As the concentration of MET was lower than that of MOR, the final volume, after mixing, may have exceeded the capacity of the smallest cassette reservoir $(50 \mathrm{~mL})$ of the initially prepared device (CADD-Legacy PCA; Smiths Medical Japan, Tokyo, Japan); therefore, it was necessary to consider the possibility of using a larger cassette $(100 \mathrm{~mL})$ than that used for MOR. The necessary volumes were sampled from OCT and MET ampoules and diluted with saline to a total volume of $101.0 \mathrm{~mL}$. The final OCT and MET concentrations were $20.8 \mu \mathrm{g} / \mathrm{mL}$ and $1.39 \mathrm{mg} / \mathrm{mL}$, respectively. Two mixtures with MET-SBS 0 and MET-SBS 1.0 were prepared. The final SBS concentrations were 0 and $0.30 \mathrm{mg} / \mathrm{mL}$, respectively. A total of $1 \mathrm{~mL}$ was transferred from each mixture to a $1.5 \mathrm{~mL}$ microtube and stored at room temperature with protection from light. The OCT concentration in each sample was then measured after 3, 7 and 10 days using HPLC.

\section{HPLC analysis}

The HPLC device (LC-2010CHT; Shimadzu Corporation, Kyoto, Japan) and analysis software (LC solution; Shimadzu Corporation, Kyoto, Japan) were used in this analysis as described previously. ${ }^{19}$ The injection volume was $10 \mu \mathrm{L}$ and detection wavelength was $210 \mathrm{~nm}$ (UV-VIS detector). Measurements were performed at room temperature using the reverse-phase column, COSMOSIL5C18-MS-II (column diameter: 4.6, column length: $150 \mathrm{~mm}$ ) (Nacalai Tesque, Kyoto, Japan). Mobile phase A (water:acetonitrile:1 M tetramethylammonium hydroxide solution $=440: 50: 10)$, mobile phase B (water:acetonitrile:1 M tetramethylammonium hydroxide solution=190:300:10) and mobile phase C (water:acetonitrile $=50: 50$ ) were prepared, and the $\mathrm{pH}$ was set to 4.5 using two types of phosphate solution $(0.17 \mathrm{M}$ sodium dihydrogenphosphate and $0.17 \mathrm{M}$ disodium hydrogenphosphate).

Using the linear gradient elution method, the mobile phase ratio was changed from $\mathrm{A}: \mathrm{B}=73: 27$ to $55: 45$ at the initiation of analysis to $12 \mathrm{~min}$, and the solution was allowed to flow at $1 \mathrm{~mL} / \mathrm{min}$ to set $\mathrm{C}$ at $100 \%$ from 12 to $17 \mathrm{~min}$. A calibration curve in the range of $0-50 \mu \mathrm{g} / \mathrm{mL}$ OCT was prepared from the peak height, and the OCT concentration was calculated from the peak height at the OCT retention time-point in each sample.

We prepared a sample at every measurement point. After storing the samples, we assayed each sample three times. For 
example, in the 'dependence on SBS concentrations' experiment, we prepared SBS at seven different concentration levels and assayed them on days $0,3,7$ and 10 . Therefore, we prepared samples of 28 measurement points 7 different level samples $\times 4$ days $=28$ samples) and assayed them three times (28 samples $\times 3$ times $=84$ points measured).

\section{Statistical analysis}

Statistical comparisons were made using a one-way ANOVA (analysis of variance) followed by Tukey's post-hoc test. Values of $\mathrm{p}<0.05$ were considered to be significant. The clinical stability of OCT was defined as maintaining at least $90 \%$ of the initial OCT concentration. ${ }^{13} 1619$

\section{RESULTS}

\section{Dependence on SBS concentrations}

When the SBS concentration was lower than $0.1 \mathrm{mg} / \mathrm{mL}$, no significant decrease was noted in the OCT concentration for 10 days after mixing with SBS at any concentration, and more than $90 \%$ of the initial OCT concentration was maintained (figure 2). However, the OCT concentration significantly decreased to less than $90 \%$ of the initial concentration on days 10,3 and 0 after mixing when the concentrations of SBS were $0.1,0.3$ and $0.5 \mathrm{mg} / \mathrm{mL}$, respectively.

\section{Compatibility study with MOR preparations}

The OCT concentration decreased significantly after storage for 10 days when OCT was mixed with 4\% MOR containing SBS; however, more than $90 \%$ was maintained (figure 3). No significant decrease was noted in any combination in the mixture with $1 \%$ MOR.

\section{Compatibility study with MET preparations}

The OCT concentration decreased significantly after storage for 3 days and thereafter when OCT was mixed with MET-SBS 1.0. The OCT level was lower than $90 \%$ of the initial concentration

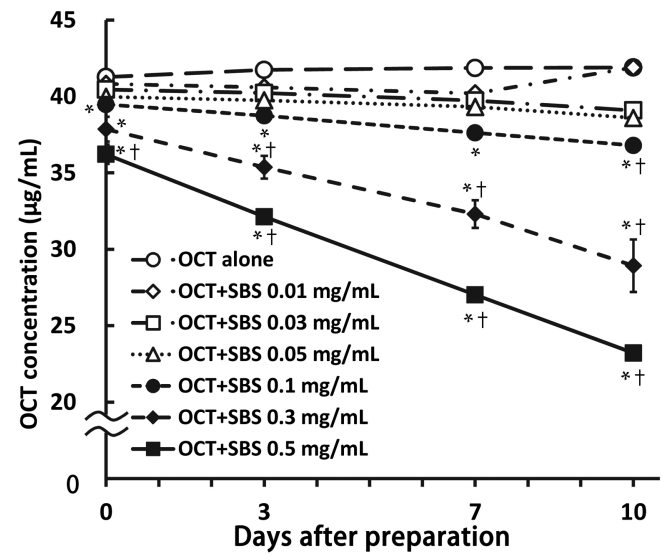

Figure 2 Time courses of octreotide (OCT) concentrations depended on sodium bisulfate (SBS) concentrations. The OCT concentration decreased with the SBS concentration and/or storage periods. Open symbols represent groups in which no significant difference was noted throughout the 10-day period. Closed symbols represent groups in which a significant difference was noted during the 10-day period. ${ }^{*} p<0.001$ vs OCT alone on each day (one-way ANOVA followed by Tukey's post-hoc test). ${ }^{\dagger}$ The percentages of OCT were less than $90 \%$ versus the calculated logical initial OCT concentrations $(41.7 \mu \mathrm{g} / \mathrm{mL})$. Values are means \pm SEM $(n=3)$. at all time-points (figure 4). By contrast, no significant decrease was noted in any combination in the mixture with MET-SBS 0.

\section{DISCUSSION}

This is the first study to clarify the dependence of OCT stability on the SBS concentration, and the following results are of clinical importance.

SBS may not influence OCT stability when its final concentration is $0.05 \mathrm{mg} / \mathrm{mL}$ or lower; it may clinically influence stability and have a negative impact at a final concentration of $0.3 \mathrm{mg} / \mathrm{mL}$ or higher. A significant decrease was observed in the concentration of OCT on day 0 , and this has been attributed to the progression of hydrolysis at the time of the measurement due to sample preparation time. Further decreases may occur in OCT concentration depending on other factors, such as the $\mathrm{pH}$, temperature and exposure to light $;^{9} 19$ therefore, mixing should be avoided under these conditions. Judgements regarding expiry dates (from immediately after preparation to the completion of its use) are varied when the final SBS concentration is $0.1 \mathrm{mg} / \mathrm{mL}$. Although OCT concentrations decreased significantly on days 0 and 3 , more than $90 \%$ of the initial concentration (theoretical value) was retained. Thus, these decreases may have no clinical influence when the preparation is used within 3 days. However, the stability of OCT may be influenced by SBS for its administration over a period that ranges from several days to a week.

Incompatibility with the SBS-containing MOR preparation was within a clinically acceptable range. SBS is only added to the $4 \%$ preparation to maintain the stability of MOR because the concentration is close to its solubility. Mixing with the $1 \%$ preparation was previously shown to be possible, but it was unclear whether it was possible with the $4 \%$ preparation because the presence of SBS had not been considered in previous compatibility studies. ${ }^{13} 2021$ Our study demonstrated that, although the OCT concentration decreased significantly on day 10, more than $90 \%$ of the initial concentration (calculated value) was retained, indicating that this decrease had no clinical

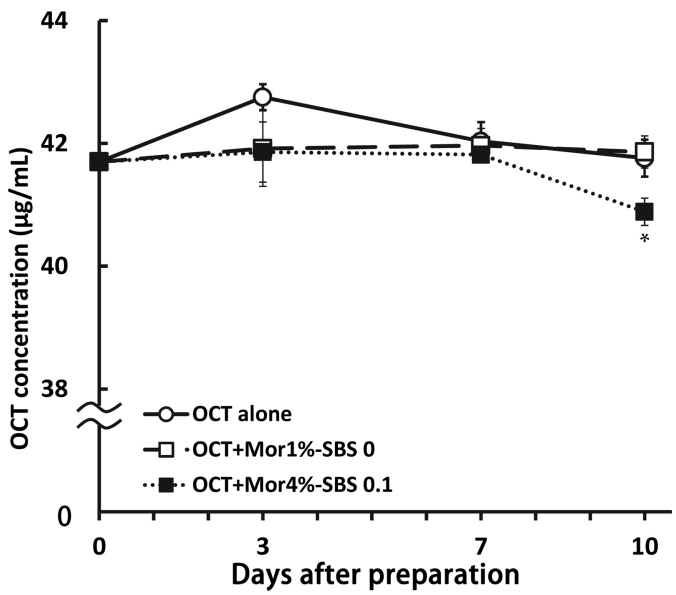

Figure 3 Octreotide (OCT) concentrations mixed with morphine (MOR) containing or not containing sodium bisulfate (SBS) for 10 days after being prepared. MOR $1 \%-S B S 0$, MOR hydrochloride injection $10 \mathrm{mg} / 1 \mathrm{~mL}$ (SBS free); MOR4\%-SBS 0.1, MOR hydrochloride injection $200 \mathrm{mg} / 5 \mathrm{~mL}$ (SBS $0.1 \mathrm{mg} / \mathrm{mL}$ ). The initial concentrations (day 0) of each group were the calculated logical values. The percentages of OCT were calculated versus the initial concentrations. OCT was decomposed after being mixed with MOR in the presence of SBS for 10 days after being prepared. * $p<0.05$ versus the OCT alone group after 10 days (one-way ANOVA followed by Tukey's post-hoc test). Values are means \pm SEM $(n=3)$. 


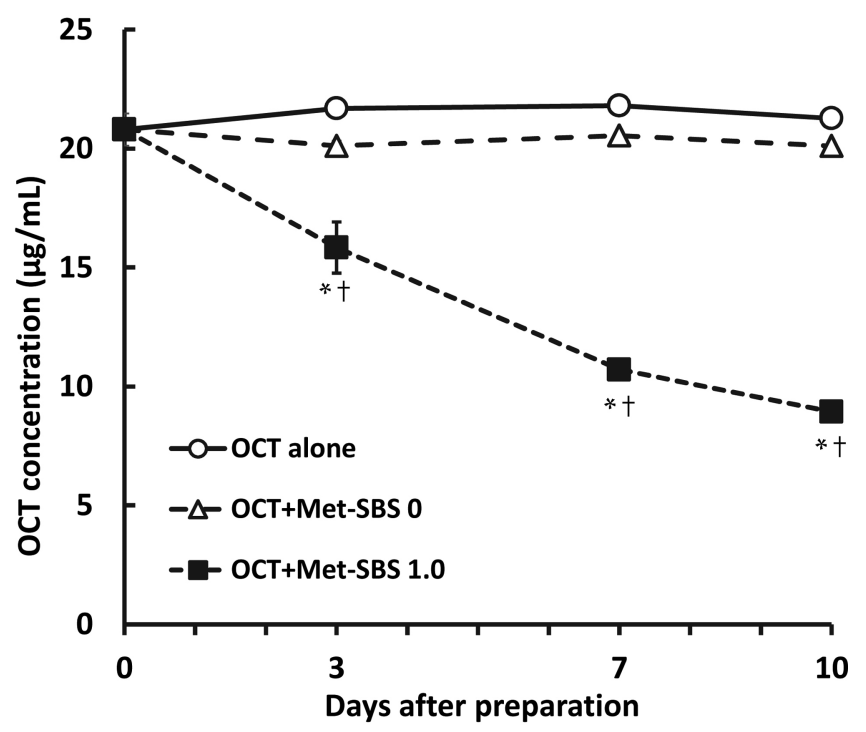

Figure 4 Octreotide (OCT) concentrations mixed with metoclopramide (MET) containing or not containing sodium bisulfate (SBS) for 10 days after being prepared. MET-SBS 0, Elieten (SBS free); MET-SBS 1.0, Primperan (SBS $1.09 \mathrm{mg} / \mathrm{mL}$ as sodium pyrosulfite $1 \mathrm{mg} / \mathrm{mL}$ ). The initial concentrations (day 0 ) of each group were the calculated logical values. The percentages of OCT were calculated versus the initial concentrations. Open symbols represent groups in which no significant difference was noted throughout the 10-day period. Closed symbols represent groups in which a significant difference was noted during the 10 -day period. * $p<0.001$ versus the OCT alone group on each day (one-way ANOVA followed by Tukey's post-hoc test). †The percentages of OCT were less than $90 \%$ versus the calculated logical initial OCT concentrations. Values are means \pm SEM $(n=3)$.

influence. The final SBS concentration in the mixture with 4\% MOR was $0.01 \mathrm{mg} / \mathrm{mL}$, and this was consistent with the results of SBS concentration dependence studies on OCT stability.

We also confirmed that mixing OCT with the SBS-containing MET preparation should be avoided even though it had previously been considered possible. Although the incompatibility of OCT with MET was reported to be non-problematic by a previous study, ${ }^{11}$ mixing has been avoided in Japan based on the results of a compatibility study performed by the Japanese OCT sales company. ${ }^{22}$ These inconsistent findings were attributed to the presence or absence of SBS. We have established a method to enable the mixing of OCT and MET, which may be of clinical significance. The rate at which the OCT concentration decreased varied among different mixing conditions; the initial concentration decreased by approximately $30 \%$ in 10 days when mixing OCT with SBS at final concentrations of $41.7 \mu \mathrm{g} / \mathrm{mL}$ and $0.3 \mathrm{mg} / \mathrm{mL}$ (figure 2), respectively, and decreased by $60 \%$ in 10 days when mixing OCT at a final concentration of $20.8 \mu \mathrm{g} / \mathrm{mL}$ with MET containing SBS, even though the final SBS concentration in both was $0.3 \mathrm{mg} / \mathrm{mL}$ (figure 4). These variations may be explained by the influence of the initial OCT concentration, storage temperature, $\mathrm{pH}$, time-dependent decreases in the SBS concentration or MET itself. In any case, it is necessary to mix OCT with MET preparations not containing SBS because the decreased OCT concentration causes problems in clinical environments.

We clarified the SBS concentration $(0.1 \mathrm{mg} / \mathrm{mL}$ or higher $)$ influencing the stability of OCT, and demonstrated it using an antiemetic and analgesic, which may be used in the clinical setting, thereby supporting our hypothesis. This has led to the framing of an important policy when selecting drugs mixed with OCT: the stability of OCT can be ensured by either selecting a formulation containing no SBS or verifying the SBS concentration; the limitation to patient activities are preventable by avoiding OCT loss and administration through another route. Staggered administration is unnecessary with mixing, thus possibly decreasing the frequency of venipuncture. Additionally, more reliable clinical studies may be needed to investigate the effects of OCT.

\section{CONCLUSION}

The stability of OCT decreased with time in an SBS concentration-dependent manner. However, this incompatibility may be clinically acceptable when the final SBS concentration is lower than $0.1 \mathrm{mg} / \mathrm{mL}$ and the mixed solution is used within 7 days. The results of the present study also indicate that the SBS concentration, as well as its presence/absence, should be considered when assessing the appropriateness of mixing OCT with other drugs. Furthermore, it may be necessary to examine the influences of the initial concentration of OCT and concentrated dextrose in order to ensure its stability when it is mixed with other drugs.

\section{What this paper adds}

What is already known on this subject

- When aggressive treatment is not an option, octreotide (OCT) is an important drug that can be used to maintain the quality of life of patients until the end stage.

- Large variations have been reported in the efficacy of OCT (44\%-100\%).

- OCT is a cyclic octapeptide whose hydrolysis is promoted by sodium bisulfate (SBS). However, the critical SBS concentration is unknown.

What this study adds

- We examined reproducibility, with a focus on the SBS concentration-dependent OCT stability of drugs made from morphine and metoclopramide, using high performance liquid chromatography.

- This study clarified the critical concentration of SBS for OCT stability.

\section{Competing interests None.}

\section{Patient consent Obtained.}

Provenance and peer review Not commissioned; externally peer reviewed.

Open Access This is an Open Access article distributed in accordance with the Creative Commons Attribution Non Commercial (CC BY-NC 4.0) license, which permits others to distribute, remix, adapt, build upon this work non-commercially, and license their derivative works on different terms, provided the original work is properly cited and the use is non-commercial. See: http://creativecommons.org/ licenses/by-nc/4.0/

\section{REFERENCES}

1 Ripamonti C, Mercadante S. Pathophysiology and management of malignant bowel obstruction. In: Hanks G, Cherny NI, Christakis NA, et al, eds. Oxford Textbook of Palliative Medicine. New York: Oxford University Press, 2010:850-63.

2 Ripamonti C, Twycross $\mathrm{R}$, Baines $\mathrm{M}$, et al. Clinical-practice recommendations for the management of bowel obstruction in patients with end-stage cancer. Support Care Cancer 2001;9:223-33.

3 Pless J, Bauer W, Briner U, et al. Chemistry and pharmacology of SMS 201-995, a long-acting octapeptide analogue of somatostatin. Scand J Gastroentero/ Supp/ 1986;119:54-64. 
4 Pless J. From somatostatin to Sandostatin: History and chemistry. Metabolism 1992;41(9 Suppl. 2):5-6.

5 Pless J. The history of somatostatin analogs. J Endocrinol Inves 2005;28(Suppl. 11):1-4.

6 Horioka M. Principles and practice of dispensing pharmacy, 2nd edn. Tokyo: Nanzando Co. Ltd, 1995;323-8 (in Japanese).

7 Cecil R, Loening UE. The reaction of the disulphide groups of insulin with sodium sulphate. Biochem J 1960;76:146-55.

8 Cecil R, Wake RG. The reactions of inter- and intra-chain disulphide bonds in proteins with sulphate. Biochem J 1962;82:401-6.

9 Tanabe K, Ohkubo J, Ikezaki T, et al. Effect of sodium bisulphate on the stability of octreotide acetate: compatibility study with dexamethasone injection. Palliat Care Res 2013:8:177-83.

10 Steger PJ, Martinelli EF, Muhlebach SF. Stability of high-dose morphine chloride injection upon heat sterilization: comparison of UV-spectroscopy and HPLC. J Clin Pharm Ther 1996;21:73-8.

11 Twycross R, Wilcock A, Mortimer J, et al. Compatibility charts. In: Palliative care formulary, 3rd edn. Nottingham: Palliativedrugs.com Ltd, 2007;583-97.

12 Mercadante S, Ferrera P, Villari P, et al. Aggressive pharmacological treatment for reversing malignant bowel obstruction. J Pain Symptom Manage 2004;28:412-16.

13 Vermeire A, Remon JP, Schrijvers D, et al. A new method to obtain and present complete information on the compatibility: study of its validity for eight binary mixtures of morphine with drugs frequently used in palliative care. Palliat Med 2002;16:417-24.
14 Shima Y, Ohtsu A, Shirao K, et al. Clinical efficacy and safety of octreotide (SMS201-995) in terminally ill Japanese cancer patients with malignant bowel obstruction. Jpn J Clin Oncol 2008;38:354-9.

15 Murakami N, Atarashi Y, Kadoya S, et al. Octreotide acetate use for patient with cancerous peritonitis and postoperative gastric cancer in home hospice care setting. Palliat Care Res 2009;4:321-9.

16 Tanabe K, Ikezaki T, Ohkubo J, et al. Efficacy criteria of octreotide in abdominal symptoms for home palliative care. Jpn J Pharm Palliat Care Sci 2010;3:53-61.

17 Yin D, Zhu Y, Xing Y, et al. Clinical observation of continuously subcutaneous-pumped octreotide infusion in palliative treatment of malignant bowel obstruction. Chin-German J Clin Oncol 2011;10:31-4.

18 Hisanaga T, Shinjo T, Morita T, et al. Multicenter prospective study on efficacy and safety of octreotide for inoperable malignant bowel obstruction. Jpn J Clin Oncol 2010;40:739-45

19 Stiles ML, Allen LV, Resztak KE, et al. Stability of octreotide acetate in polypropylene syringes. Am J Hosp Pharm 1993;50:2356-8.

20 Fielding $\mathrm{H}$, Kyaterekera N, Skellern GG, et al. The compatibility and stability of octreotide acetate in the presence of diamorphine hydrochloride in polypropylene syringes. Palliat Med 2000;14:205-7.

21 Mercadante $S$. Tolerability of continuous subcutaneous octreotide used in combination with other drugs. J Palliat Care 1995;11:14-16.

22 Novartis Pharma Co. Ltd., eds. The Drug Interview Form of Sandostatin for s.c. Injection. Tokyo: Novartis Pharma Co. Ltd. 2009;48-50. 